\title{
Vertical Pelvic Fracture with Hemodynamical Stability
}

\author{
Dong Hun Kim', Hee-Gon Park ${ }^{2}$ \\ 'Department of Surgery, Trauma Center, Dankook University Hospital, Cheonan, Korea \\ ${ }^{2}$ Department of Orthopedic Surgery, Dankook University College of Medicine, Cheonan, Korea
}

Vertically unstable fractures of the pelvic ring, mostly caused by falls from heights or traffic accidents, are high-energy injuries with high mortality and morbidity rate. Patients with unstable pelvic ring fracture usually have hemodynamic instability, presenting a higher ratio of patients in shock already in the field. We described a case in which initial conservative treatment including fluid resuscitation and pelvic traction for vertical pelvic fracture with stable hemodynamics after a blunt trauma.

Key Words: Pelvic fracture; Unstable; Hemodynamics

(Trauma Image Proced 2017(2):72-74)

\section{CASE}

A 46-year-old man was ran over by an excavator and admitted to the emergency department. Upon arrival, he was alert with transient hypotension having a response to the transfusion of 2 units of packed red blood cells. The injury severity score was 29. The Focused Assessment with Sonography for Trauma showed no intraabdominal fluid collection. Supine pelvic X-ray showed multiple fractures in left pelvic with a dislocation (Fig. 1). Abdomen computed tomography scan revealed comminuted fractures and a vertical dislocation of left sacral ala and left pubic ramus with diastatic widening of left sacroiliac joint and hematoma around pelvic fractures without extravasation (Fig. 2). His stable hemodynamics was preserved without invasive procedures including angiographic embolization and massive transfusion. External pelvic fixation with C-clamp was performed the

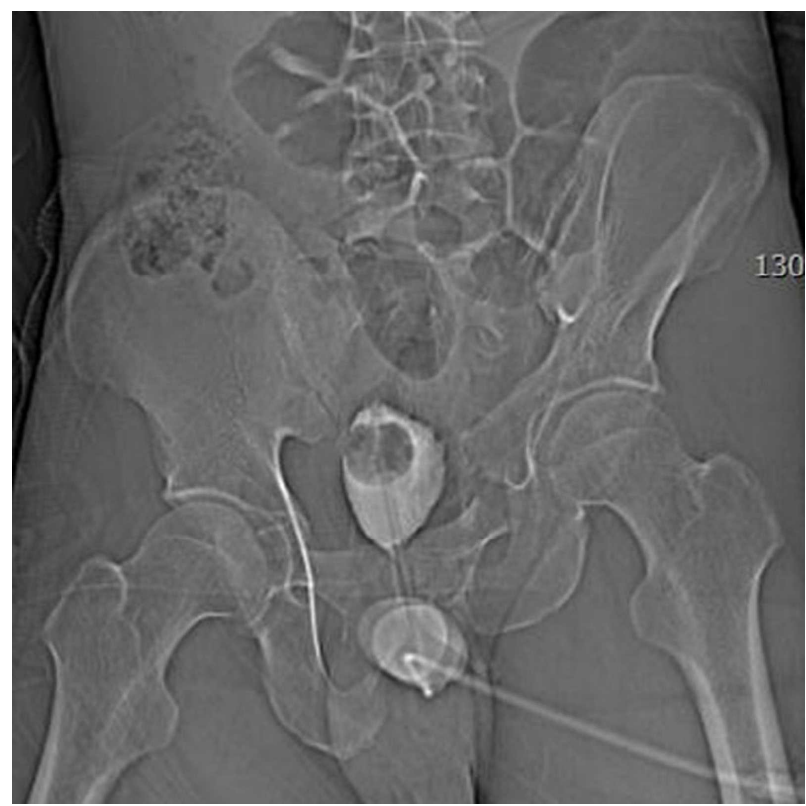

Fig. 1. Supine pelvis radiography shows multiple pelvic fracture with left sacroiliac joint injury. next day after skeletal traction applied to the left pelvic

Received: April 19, 2017 Revised: June 14, 2017 Accepted: June 16, 2017

Correspondence to: Dong Hun Kim, Department of Surgery, Trauma Center, Dankook University Hospital, 201 Manghyang-ro, Dongnam-gu, Cheonan, Chungnam 31116, Korea

Tel: 82-41-550-7661, Fax: 82-41-550-0039, E-mail: saint7331@gmail.com

Copyright (c) 2017 Korean Association for Research, Procedures and Education on Trauma. All rights reserved.

(c) This is an open-access article distributed under the terms of the Creative Commons Attribution Non-Commercial License (http://creativecommons.org/ licenses/by-nc/4.0) which permits unrestricted noncommercial use, distribution, and reproduction in any medium, provided the original work is properly cited 

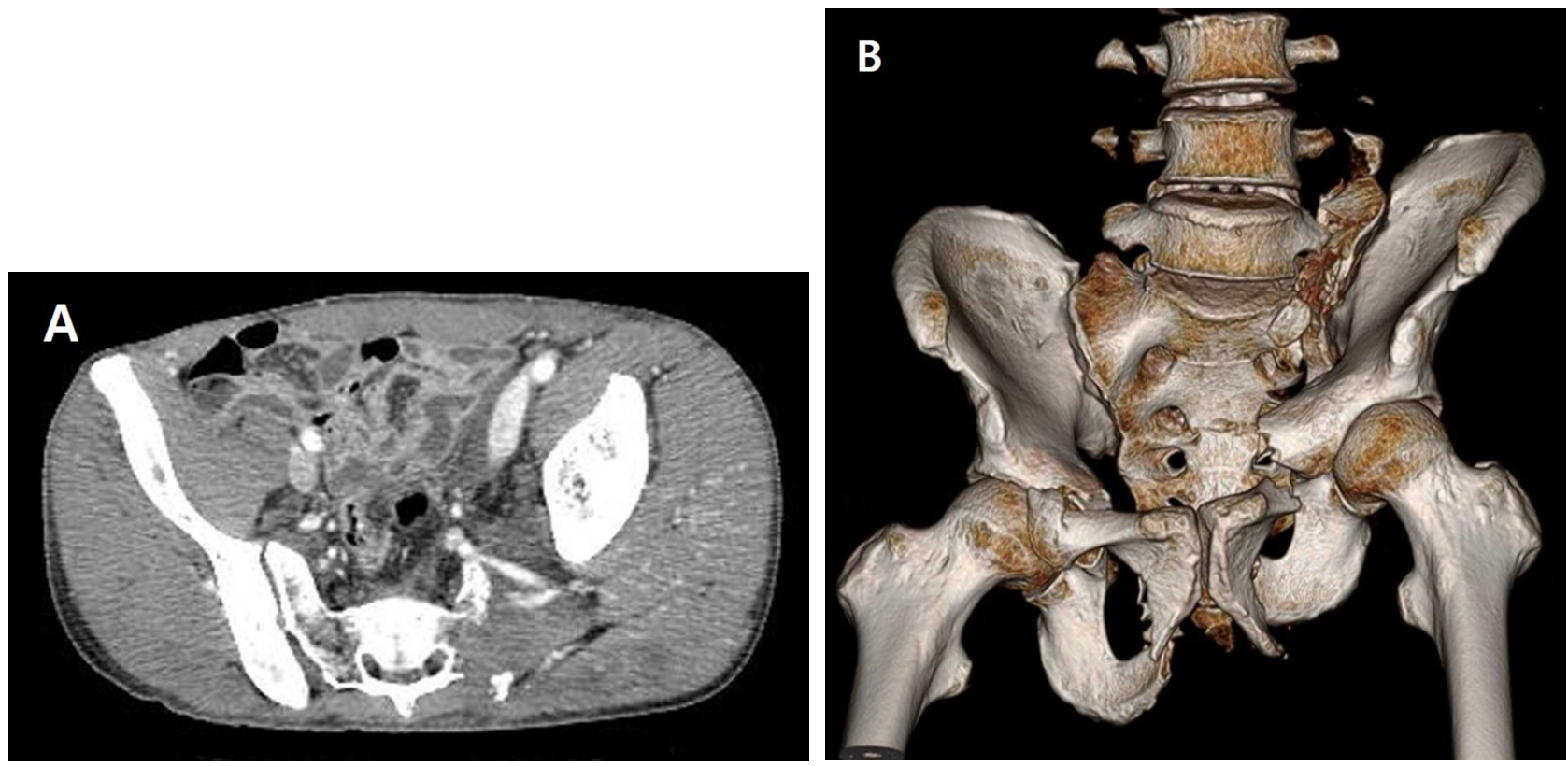

Fig. 2. (A) Abdomen computed tomography scan shows retroperitoneal hematoma of pelvis around left sacroiliac joint without extravasation, and (B) 3-D reconstruction of pelvis shows comminuted fractures and vertical dislocation of left sacroiliac joint, and bilateral fractures of pubic superior and inferior ramus.
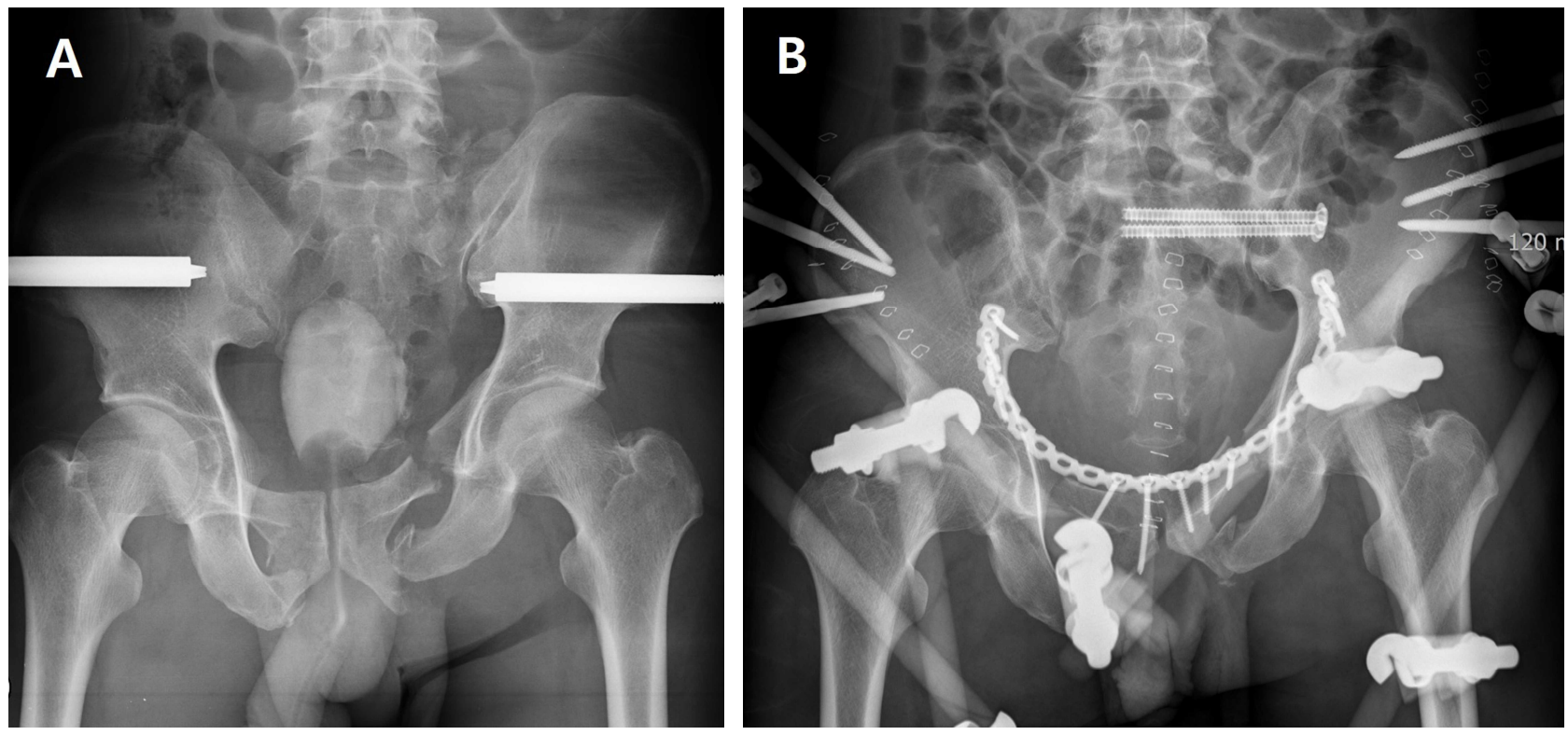

Fig. 3. Supine pelvis radiography shows (A) external fixation of sacroiliac joint with C-clamp on hospital day 2, and (B) conversion with internal and external fixation of pelvis in hospital day 7 .

bone and lower extremity on admission day (Fig. 3A). Then, closed reduction with internal fixation of pelvis and anterior external pelvic fixation was performed in hospital day 7 (Fig. 3B).

\section{DISCUSSION}

The injury pattern of vertical pelvic fracture was rotationally and vertically unstable, with a complete disruption of the posterior osseous ligamentous structure 
of the pelvis (1). Patients with unstable pelvic ring fracture, Young-Burgess classification types $\mathrm{B}$ and $\mathrm{C}$ showed significantly worse vital signs compared with type A, presenting a higher ratio of patients in shock already in the field for them (2). This case was an unstable pelvic ring fracture, Young-Burgess classification type $\mathrm{C}$, but hemorrhagic shock did not develop, not based on its injury severity. Initial resuscitative management was performed without massive transfusion, and operative management was carried out next day during the skeletal traction. Therefore, resuscitative treatment for pelvic fracture should be determined based on the hemodynamics and bleeding tendency of patient, with even unstable pelvic ring fracture.

\section{Conflict of Interest Statement}

No potential conflict of interest relevant to this article was reported.

\section{REFERENCES}

1. Griffin DR, Starr AJ, Reinert CM, Jones AL, Whitlock S. Vertically unstable pelvic fractures fixed with percutaneous iliosacral screws: does posterior injury pattern predict fixation failure? J Orth Trauma. 2002;17(6):399-405.

2. Burkhardt $M$, Nienaber $U$, Pizanis $A$, Maegele $M$, Culemann U, Bouillon B, et al. Acute management and outcome of multiple trauma patients with pelvic disruptions. Crit Care. 2012;16(4):R163. 